\title{
Introduction of a comprehensive management protocol for severe sepsis is associated with sustained improvements in timeliness of care and survival
}

\author{
R MacRedmond, ${ }^{1}$ K Hollohan, ${ }^{2}$ R Stenstrom, ${ }^{2}$ R Nebre, ${ }^{2}$ D Jaswal, ${ }^{1}$ P Dodek ${ }^{3}$
}

${ }^{1}$ Division of Critical Care Medicine, Department of Medicine, University of British Columbia, Vancouver, British Columbia, Canada ${ }^{2}$ Department of Emergency Medicine, University of British Columbia, Vancouver, British Columbia, Canada ${ }^{3}$ Division of Critical Care Medicine, Department of Medicine, University of British Columbia and Center for Health Evaluation and Outcome Sciences, St Paul's Hospital, Vancouver, British Columbia, Canada

\section{Correspondence to}

Ruth MacRedmond, UBC James Hogg Research Centre, St Paul's Hospital, Rm 166-1081 Burrard Street, Vancouver, BC, Canada V6Z1Y6;

rmacredmond@mrl.ubc.ca

Accepted 27 August 2009

Published Online First

29 July 2010

\begin{abstract}
Introduction Mortality from severe sepsis can be improved by timely diagnosis and treatment. This study investigates the effectiveness of a comprehensive management protocol for recognition and initial treatment of severe sepsis that spans from the emergency department (ED) to the intensive care unit. Methods Interventions included development of a management algorithm including early goal-directed therapy, a computerised physician order entry set for suspected sepsis, introduction of invasive haemodynamic monitoring and antibiotics stocked in the $E D$, and an extensive education campaign involving ED nurses and physicians.
\end{abstract}

Main results In the 6 months after introduction of the protocol, 37 patients who had severe sepsis were identified in the ED. Compared to a randomly selected group of 37 patients who had severe sepsis and who were transferred directly to the intensive care unit before introduction of the protocol, significant improvements were observed in mean time to initiation of early goaldirected therapy (3.2 vs $10.4 \mathrm{~h}, \mathrm{p}=0.001$ ) and to achievement of resuscitation goals (10.4 vs $30.1 \mathrm{~h}$, $p=0.007)$. There was a trend towards more rapid administration of antibiotics ( 1.4 vs $2.7 \mathrm{~h}, \mathrm{p}=0.06$ ). This was associated with a decrease in crude hospital mortality rate from $51.4 \%$ to $27.0 \%$ (absolute risk reduction $=24 \%, 95 \% \mathrm{Cl} 3 \%$ to $47 \%$ ). Improvements were sustained in the follow-up audit at 16 months.

Conclusions Introduction of a comprehensive management protocol to address early recognition and management of severe sepsis in the ED is associated with sustained improvements in processes of care.

Severe sepsis is a complex syndrome comprising a range of clinical conditions caused by the body's systemic response to an infection resulting in single or multiple organ dysfunction or failure. There are 750000 cases of severe sepsis annually in North America. It is a major cause of mortality, killing approximately 1400 people worldwide every day. 12

Recent years have seen a revolution in the diagnosis and treatment of sepsis, with the development of operational definitions for severe sepsis and septic shock ${ }^{3}$ and application of best evidence to define standards of care for this complex disease. In 2004, the Surviving Sepsis Campaign issued guidelines for the comprehensive management of severe sepsis (updated in $2008^{4}$ ) with the aim of reducing sepsis mortality by $25 \%$ in 5 years ${ }^{5}$. These guidelines were endorsed by the Institute for Healthcare Improvement (IHI), who translated them into resuscitation (early), and management (late) "bundles", or grouped interventions. Although patients who have severe sepsis generally require admission to an intensive care unit (ICU), they frequently present to the emergency department (ED), where prompt diagnosis and treatment can significantly improve outcome.

In an effort to implement evidence-based recommendations for the management of severe sepsis, we developed a sepsis protocol as a collaborative quality improvement initiative between the ED and ICU at our hospital. We focused on the impact of process changes in the management of patients who had severe sepsis in the ED by ED staff, with support from ICU staff as required, up to the time of discharge from the ED to the ICU or ward. The purpose of this study was to evaluate the impact of this quality improvement (OI) initiative on key processes and outcomes for these patients.

\section{METHODS}

The study was approved by the research ethics board of Providence Health Care.

\section{Assessment of the problem}

St Paul's Hospital is a tertiary care teaching hospital in downtown Vancouver, Canada, that has 500 beds, a "closed" medical-surgical ICU of 15 beds staffed by dedicated intensivists, and an ED that serves $>60000$ patients per year and that is staffed by dedicated and certified emergency physicians. Care in both areas is delivered by housestaff who are supervised by staff physicians.

We convened a multidisciplinary team that consisted of physicians and nurse educators from the ED and ICU and members of the quality and utilisation management team at our hospital. The team met bimonthly over an 8-month period to address several issues related to the care of patients who have severe sepsis or septic shock (figure 1). Principles of lean thinking were applied to the initial examination of the care path or value stream of septic patients after arrival in the ED. ${ }^{6}$ The process was thus focused on eliminating "non-value added" steps such as waiting for antibiotics to be delivered from the pharmacy and improving the flow of "value added" steps such as facilitating invasive haemodynamic monitoring. 
Figure 1 Flow diagram of quality improvement process.

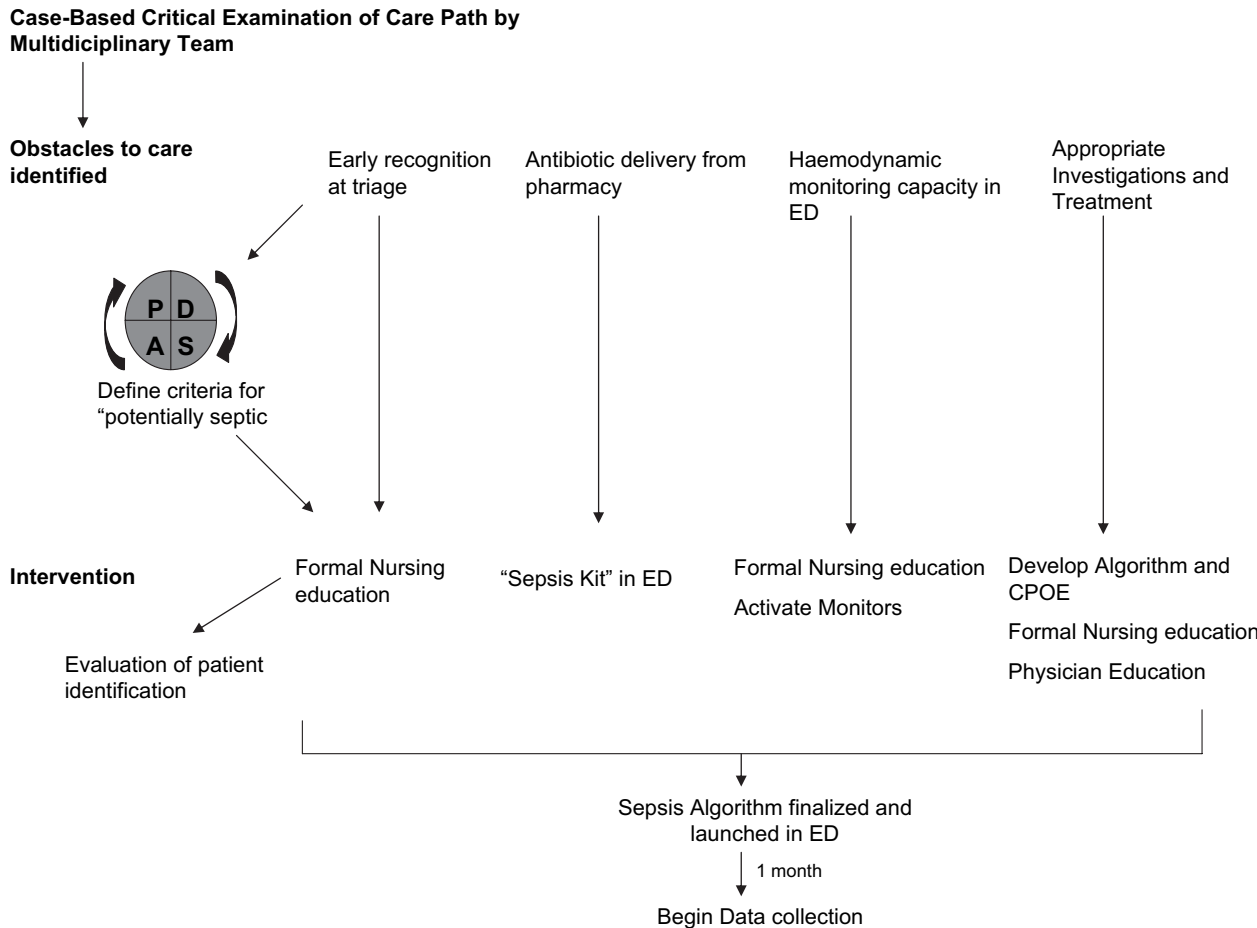

\section{Interventions}

\section{Education of staff}

A series of plan-do-study-act cycles were used to determine the optimum method of screening for suspected sepsis at triage. This process established the criteria for "potential sepsis" as suspected or proven infection plus the presence of two or more systemic inflammatory response syndrome (SIRS) criteria (of those criteria that were identifiable at triage: temperature $>38^{\circ}$ or $<36^{\circ} \mathrm{C}$; heart rate $>90$; respiratory rate $>20$ ) or altered sensorium. Based on experiences of other centres (Dr E Rivers, personal communication) nurses were also encouraged to activate the sepsis protocol for any patients in whom they suspected severe sepsis based on other elements of their clinical assessment.

All 86 ED nurses attended one of ten 4-h education sessions over a 6-week period. Attendance was mandatory and paid. The session began with a theoretical lecture by the ICU/ED physicians that focused on the concept of sepsis as a disease entity, early recognition of "potential sepsis" (using case-based examples) and introduction of the sepsis algorithm (figure 2). This was followed by practical instruction and demonstration of haemodynamic monitoring setup by the ICU and ED educators. Nurses then went to the ICU where they were "buddied" with an ICU nurse for further practise.

Before launching the protocol, a pilot study was done to determine the effect of these educational sessions on accuracy of patient identification at triage. Fifteen trained triage nurses were asked to categorise consecutive ED patients as "potentially septic" or not (272 patients), and this process was repeated with the same nurses 2 to 4 weeks after each nurse attended a sepsis education session (198 patients). Accuracy of responses was independently evaluated by one of three physicians who retrospectively reviewed the appropriate medical records.

ED physicians and residents were introduced to the protocol at Grand Rounds. The effect of physician education was not formally assessed.

\section{Sepsis algorithm}

The sepsis algorithm was developed to encourage a standardised, chronological approach to the septic patient based on current best evidence and the recommendations of the Surviving Sepsis Campaign $^{5}$ (figure 2). The algorithm was posted prominently throughout the ED.

\section{Order set}

A computerised physician order entry set was developed using the Eclipsys Sunrise Clinical Manager system (Eclypsis Corporation, Atlanta, GA, USA) This order set included initial investigations to determine the site of infection and potential organ dysfunction, rapid intravenous fluid infusions, vasoactive drugs and antibiotics (broad spectrum based on suspected site and patient risk factors). A supply of those antibiotics recommended in the order sets was made available in the ED (sepsis kit). The sepsis algorithm was activated by the triage nurse who initiated appropriate investigations, fluid resuscitation and physician review within 15 min. Further orders were initiated by the physician. Patients who subsequently were found not to have severe sepsis or septic shock did not complete the protocol.

\section{Invasive haemodynamic monitoring}

Invasive central venous pressure and invasive blood pressure monitoring was made available in two high-intensity areas in the ED. ED physicians were encouraged to insert the central venous catheter, with support from the ICU team when required.

\section{Measures of change}

\section{Study population}

After a 1-month run-in period to familiarise staff with the protocol, data were collected for 6 months. Patients were screened from the following groups: all patients in whom the ED sepsis order set was activated, patients who had a serum lactate ordered at any time in their hospital stay, patients who had a hospital discharge diagnosis of sepsis or infection and all patients admitted to the ICU during the study period. Patients were included in the analysis if they fulfilled all of the following criteria: admission via ED and diagnosis of severe sepsis based on suspected or proven infection plus any of hypotension, 


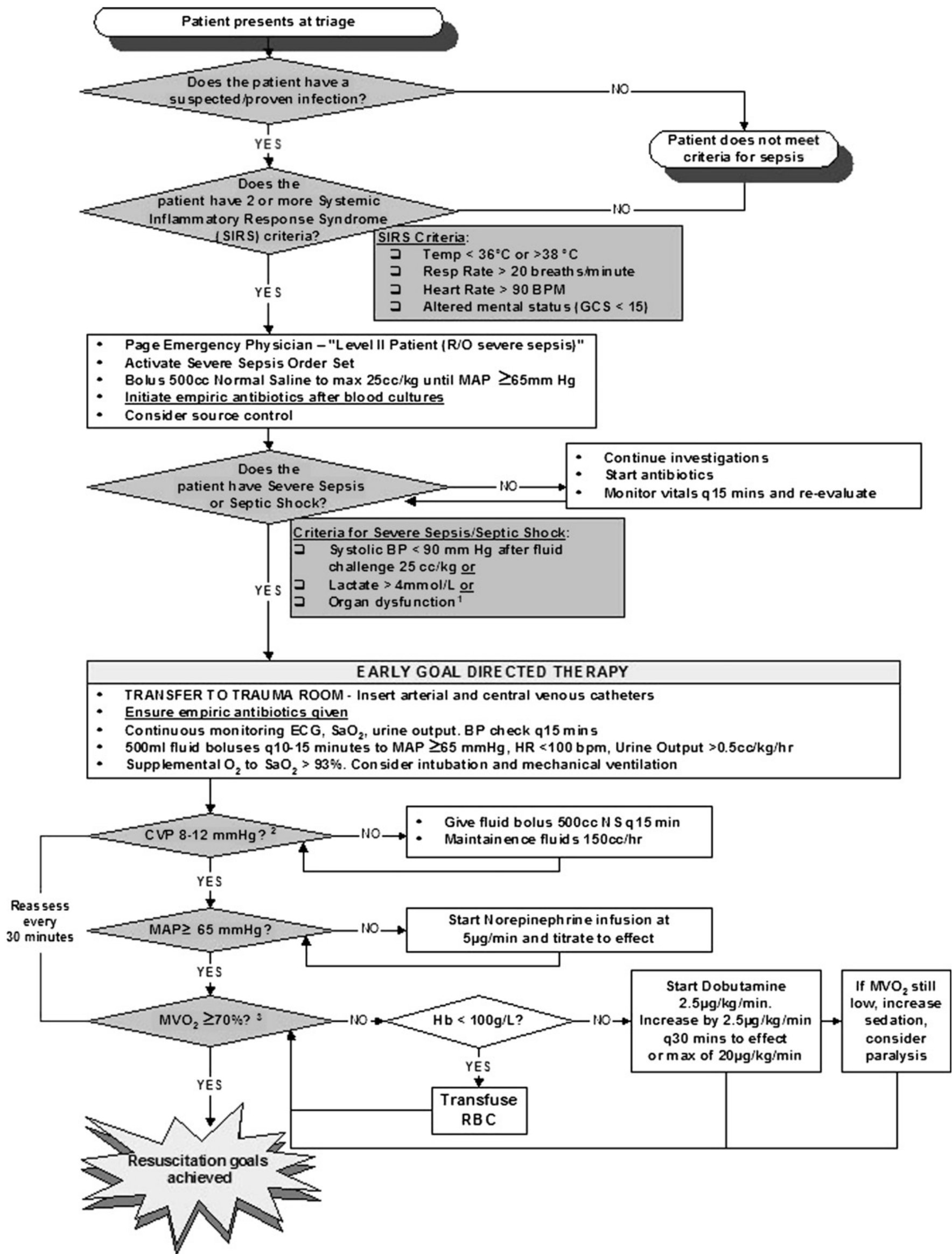

Figure 2 Management algorithm for patients presenting to the ED with suspected sepsis. CVP, central venous pressure; MAP, mean arterial pressure; $\mathrm{MVO}_{2}$, mixed venous oxygen saturation. 
Table 1 Demographics of the patient population

\begin{tabular}{llll}
\hline & Control & Protocol & p Value \\
\hline Total & 37 & 37 & \\
\% male & 67.6 & 67.6 & \\
Age (years), mean (SD) & $52.6(17.7)$ & $45.1(13.8)$ & 0.07 \\
APACHE II, mean (SD) & $24.9(6.8)$ & $22.6(8.1)$ & 0.26 \\
Site of infection & & & \\
$\quad$ Lung & 21 & 26 & 0.23 \\
Abdomen & 3 & 2 & 0.65 \\
Endocarditis & 3 & 4 & 0.69 \\
Skin & 4 & 5 & 0.72 \\
Urinary tract & 1 & 4 & 0.17 \\
CNS & 2 & 0 & 0.16 \\
Line & 0 & 1 & 0.32 \\
No site & 5 & 6 & 0.75 \\
Comorbidities & & & \\
HIV & 17 & 14 & 0.48 \\
Hepatitis B/hepatitis C & 24 & 16 & 0.06 \\
Chronic renal failure & 4 & 3 & 0.69 \\
Chronic liver failure & 3 & 2 & 0.65 \\
CHF & 4 & 3 & 0.69 \\
DM & 7 & 5 & 0.53 \\
COPD & 3 & 4 & 0.69 \\
Transplant & 1 & 1 & 1.00 \\
\hline APACHEIIAcHe & &
\end{tabular}

APACHE II, Acute Physiology and Chronic Health Evaluation II; CNS, central nervous system; $\mathrm{DM}$, diabetes mellitus.

hyperlactatemia $(>4 \mathrm{mmol} / \mathrm{l}$ ) or organ dysfunction after an initial fluid resuscitation of at least $25 \mathrm{ml} / \mathrm{kg}^{3}$ A historical control group of equal number was randomly selected from the ICU database of 98 patients who were admitted from the ED to the ICU because of severe sepsis during the period June 2003 to July 2004 (table 1).

The start time for all process measures was the time that the patient met criteria for severe sepsis or septic shock. Time of initiation of early goal-directed therapy (EGDT) was recorded as the time when the central venous catheter was inserted. Time of receipt of antibiotics was recorded as time of when the last of all appropriate protocol antibiotics began infusing as recorded in the nursing notes. Time of resuscitation targets met was recorded as the time when all of the targets of mean arterial pressure $>65 \mathrm{~mm} \mathrm{Hg}$ and central venous oxygen saturation $>70 \%$ with a CVP of at least $8 \mathrm{~cm} \mathrm{H}_{2} \mathrm{O}$ were recorded in the nursing record.

The primary goal of this quality improvement initiative was to reduce hospital mortality in patients who had severe sepsis
Table 2 Outcome of the two patient populations

\begin{tabular}{|c|c|c|c|}
\hline & Control \% & Protocol \% & $\overline{\text { p Value }}$ \\
\hline Crude mortality & 51.4 & 27.0 & 0.02 \\
\hline \multicolumn{4}{|c|}{ Predicted mortality (APACHE) } \\
\hline Mean $(95 \% \mathrm{Cl})$ & 47.8 (39.6 to 55.9 ) & 42.9 (34.4 to 51.3$)$ & 0.50 \\
\hline $\begin{array}{l}\text { Mortality-ICU patients } \\
\text { data complete }\end{array}$ & 51.5 & 29.0 & \\
\hline $\begin{array}{l}\text { Standardised mortality } \\
\text { ratio }(95 \% \mathrm{Cl})\end{array}$ & $1.1(0.7$ to 1.6$)$ & $0.7(0.4$ to 1.2$)$ & 0.19 \\
\hline \multicolumn{4}{|l|}{$\begin{array}{l}\text { ICU length of } \\
\text { stay-survivors (days) }\end{array}$} \\
\hline Median (I0 range) & 8.0 (3.5 to 11.0$)$ & $7.0(2.0$ to 11.5$)$ & 0.87 \\
\hline
\end{tabular}

APACHE, Acute Physiology and Chronic Health Evaluation II; ICU, intensive care unit; IQ, interquartile.

and were admitted via the ED by $10 \%$ in 6 months. Process measures were based on the IHI resuscitation bundle and were defined as measurement of serum lactate in all patients, drawing of blood cultures before administration of antibiotics, administration of broad-spectrum antibiotics within $1 \mathrm{~h}$ and achievement of resuscitation goals within $6 \mathrm{~h}$.

\section{Measurement of sustained effect}

We audited the management of severe sepsis and septic shock in the ED 10 months after the end of the previous study period using the same methods. No further formal education sessions or process changes were done between these observation periods.

\section{Statistics}

Data are summarised as median (interquartile range) for variables that are skewed and mean (SD) for normally distributed data. Unpaired Student's t test, sign test or $\chi^{2}$ test was used as appropriate for testing of differences between groups. Calculations of the exact confidence limits for the standardised mortality ratios (SMRs) were based on the relation between the Poisson distribution and the $\chi^{2}$ distribution. ${ }^{7}$ Unpaired t test was used to test for the significant difference between the SMRs of the groups. Statistical software Graphpad Prism 3 was used for the analysis.

\section{RESULTS}

After the educational sessions, every nurse improved in the identification of septic patients ( $i g n$ test $Z=-3.1 ; p=0.002$ ). Sensitivity for the identification of potentially septic patients improved from $75 \%$ before the education sessions to $92.3 \%$
Figure 3 Time to administration of antibiotics. Data are run charts of individual patients with mean and upper $99.7 \% \mathrm{Cl}$ for control and protocol groups.

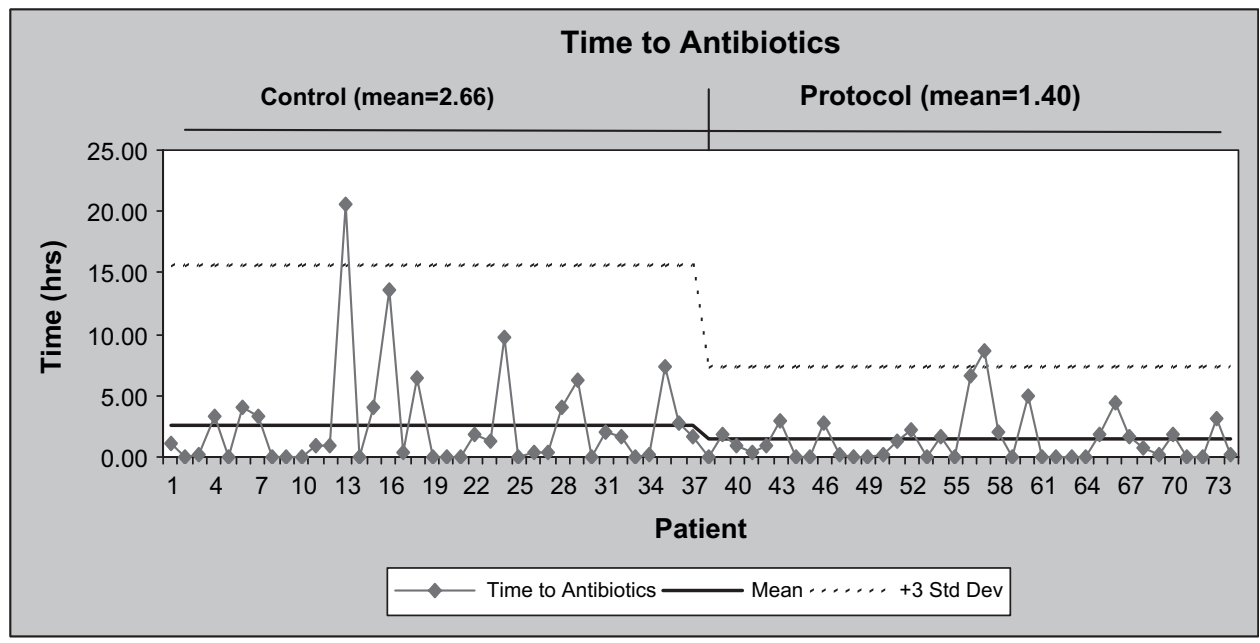


Figure 4 Time to initiation of early goal-directed therapy. Data are run charts of individual patients with mean and upper $99.7 \% \mathrm{Cl}$ for control and protocol groups.

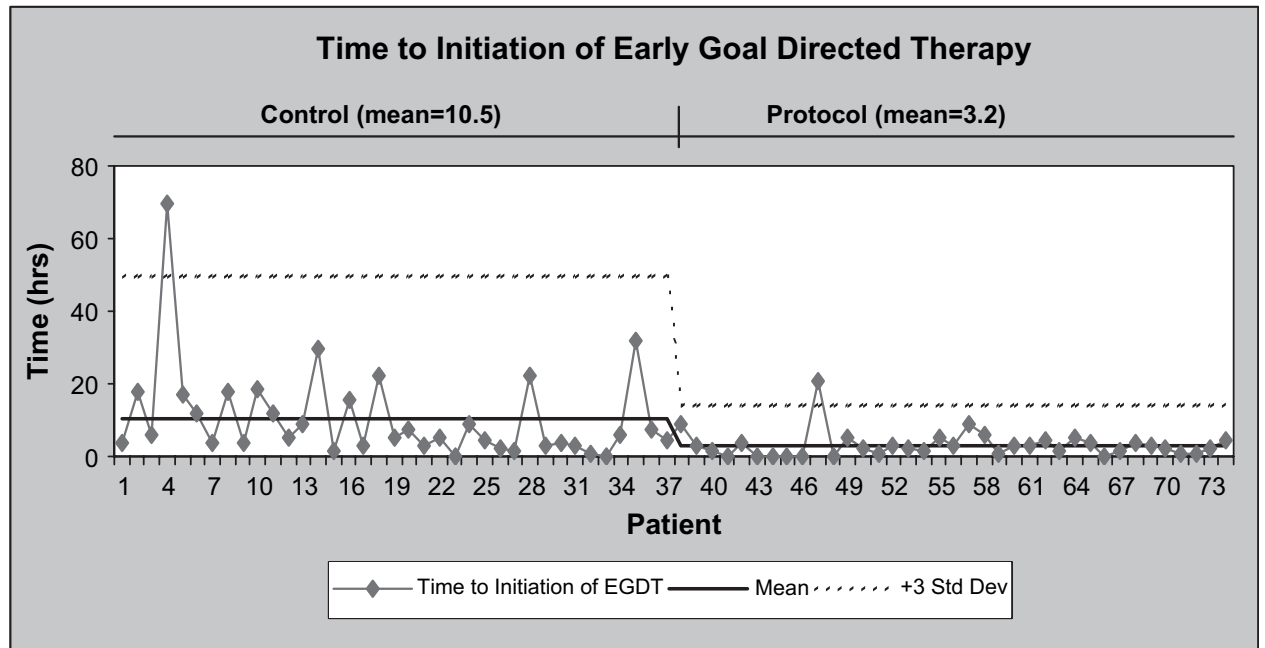

afterwards $\left(\chi^{2}(1 d f)=22.4 ; p<0.001\right)$. Specificity of the assessment was not significantly different between the two sessions: $91.1 \%$ before and $90.1 \%$ after $\left(\chi^{2}(1 d f)=0.43 ; p=0.84\right)$.

Unadjusted hospital mortality was significantly lower in the post-protocol patients compared with the retrospectively collected control population (table 2) (absolute risk reduction (ARR) $24 \%$, 95\% confidence interval (CI) $3 \%$ to 47\%). To adjust for differences in expected mortality, we examined SMRs using hospital mortality predicted by Acute Physiology and Chronic Health Evaluation II (APACHE II) score. Because APACHE II is validated only in critically ill patients admitted to the ICU, patients who were resuscitated and admitted to the general medical floor from the ED (6 of 37 patients) were not included in this analysis; in addition, data were incomplete for 4 of the 37 historical control patients. There was a large and clinically significant reduction in the SMR after introduction of the protocol, although this did not reach statistical significance.

After introduction of the sepsis protocol, time to administration of antibiotics decreased (figure 3), EGDTwas initiated more promptly (figure 4) and resuscitation goals were met more quickly (figure 5). The percentage of patients who had resuscitation goals met within $6 \mathrm{~h}$ was $62.2 \%$ in the post-protocol group compared with $13.5 \%$ in the pre-protocol group $(p<0.0001)$. There was also a reduction in the standard deviation of all three process measures in the post-protocol cohort, indicating that variability in practise was also reduced after introduction of the protocol.

Figure 5 Time to achievement of resuscitation goals. Data are run charts of individual patients with mean and upper $99.7 \% \mathrm{Cl}$ for pre-protocol and postprotocol groups.
Lactate levels were recorded in all patients in the post-protocol group and in 34 of 37 of those in the control group. The high measurement rate in the pre-protocol group reflects the fact that all of these patients were admitted to the ICU. Compliance with obtaining blood cultures before administration of antibiotics was $92.1 \%$ post protocol.

Improvements in process measures and outcomes were improved or sustained 10 months after the first data collection period (table 3). A total of 46 patients who had severe sepsis were identified during the second study period; 37 of these were admitted to the ICU. The increase in the total number of eligible patients likely reflects an outbreak of invasive pneumococcal disease that occurred in the downtown area served by St Paul's Hospital during this period. Of the 37 patients admitted to ICU, average APACHE II score was 22.2 (20.1 to 24.1), which was not significantly different from the control or first study period groups previously analysed. Crude mortality was $21.7 \%$ and SMR by APACHE was 0.65 (0.36 to 1.11$)$.

\section{LESSONS AND MESSAGES}

\section{Clinical impact of the management protocol}

By converting existing evidence into a simple algorithm, raising awareness of sepsis with an extensive education campaign and facilitation of care through a number of key interventions, we achieved clinically significant improvements in survival that were sustained up to 1 year after the initial study period.

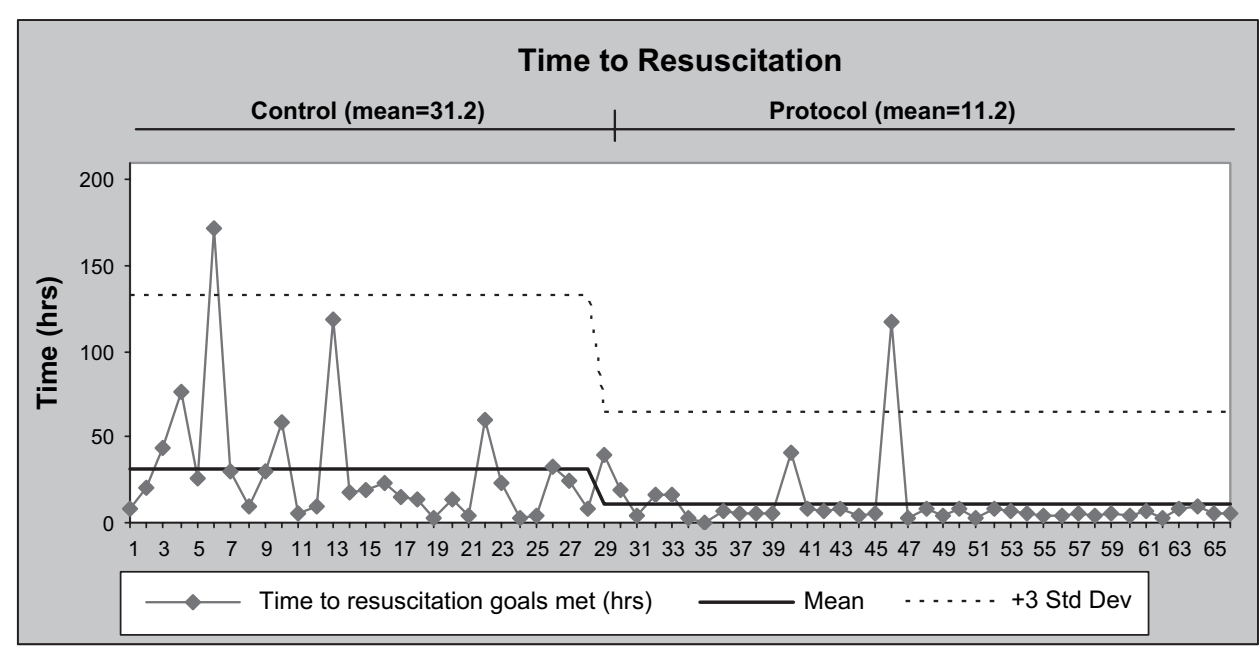


Table 3 Comparison of process of care outcomes in follow-up audit. Data are median (IQR). P values are compared to control group.

\begin{tabular}{llll}
\hline & Control & Protocol & Follow-up \\
\hline Time to antibiotics & $1.0(0$ to 3.6$)$ & $\begin{array}{l}0.4(0 \text { to } 1.9) \\
\mathrm{p}=0.06\end{array}$ & $\begin{array}{l}0.3(0 \text { to } 1.6) \\
\mathrm{p}=0.01\end{array}$ \\
& & $\begin{array}{l}2.6(0.8 \text { to } 4.1) \\
\mathrm{p}=0.001\end{array}$ & $\begin{array}{l}3.2(2.0 \text { to } 5.8) \\
\mathrm{p}=0.004\end{array}$ \\
Time to initiation of EGDT & $5.4(3.2$ to 16.4$)$ & $\begin{array}{l}5.3(4.5 \text { to } 8.4) \\
\mathrm{p}=0.007\end{array}$ & $\begin{array}{l}6.7(3.3 \text { to } 12.6) \\
\mathrm{p}=0.0006\end{array}$ \\
\hline $\begin{array}{l}\text { Time to achievement } \\
\text { of resuscitation goals }\end{array}$ & $19.2(2.8$ to 36.1$)$ &
\end{tabular}

\section{Which interventions made the difference?}

As with any study of the effect of a protocol, it is extremely difficult to identify which process(es) were responsible for the observed reduction in mortality. After the education sessions, we found significant improvement in the early identification of patients who had potential sepsis, and we believe that increased awareness of the time-critical nature of sepsis treatment among ED nurses and physicians was key to the successful implementation of the protocol.

Although we used all of the elements of the IHI resuscitation bundle in our protocol, we elected not to measure and report on compliance with the various interventions in this way. It remains unclear from published evidence whether compliance with the complete bundle will confer a survival advantage superior to any one or a combination of the elements. We wished to avoid negative implications of "failing" the bundle because of failure to complete one element of what might otherwise be an excellent resuscitation effort. Although audit of care bundles have been suggested as better indicators of quality of care than traditional outcome measures, ${ }^{8}$ positive feedback of our successes effected enthusiasm for adoption of change and, in our opinion, improved compliance with the protocol.

Our protocol included only those elements of the IHI resuscitation bundle that were time-critical and thus most pertinent to the ED care of patients who have severe sepsis. In contrast to other studies, 910 we did not include elements of the "late" management bundle that were already standard of care in our ICU during both baseline and study periods. Although other studies examined EGDT alone, ${ }^{11}$ this study focuses on all elements of the early intervention, including initial recognition and prompt antibiotic administration.

\section{Empowering change within the ED-collaboration and champions}

Other protocols have used a "sepsis team" as a type of medical emergency team for the care of patients who have severe sepsis in the ED. ${ }^{9}$ We instead adopted a collaborative model to empower the ED staff in the diagnosis and early management of severe sepsis. Key elements were championing of the protocol by senior ED physicians and a commitment from the ICU team to ongoing care of the patients in the ED and timely transfer of patients to the ICU.

\section{Barriers to change}

Our experience with regard to barriers to improvement were similar to those of other institutions, ${ }^{12}{ }^{13}$ namely, recognition of sepsis as a clinical entity and identification of septic patients, resistance to changes in practice and introduction of invasive haemodynamic monitoring. A frequently voiced concern was that the protocol would result in a delay in transfer of patients out of the ED to the ICU, thus "blocking" the high-intensity area in the ED. Our collaborative model allowed this resistance to be overcome. Supporters in the ED focused on the necessity of delivering the highest quality of care in their department, whereas ICU staff committed to transferring patients as early as was feasible. After introduction of the protocol, patients did remain longer in the $\mathrm{ED}$; however, most of the patients $(65 \%)$ achieved their resuscitation goals there.

Problems identified by other groups regarding the calibration and tracking of continuous central venous oxygen saturation ${ }^{13}$ were obviated by the use of intermittent sampling rather than continuous monitoring and allowed us to achieve our resuscitation goals within the desired 6-h time frame.

\section{LIMITATIONS}

The major limitation to this study is the use of a retrospectively collected control population randomly selected from the ICU database and the before-after study design. This before-after approach was the only one available to us because we felt it would be unethical to randomise patients when many of the protocol objectives (timely antibiotics, EGDT, etc) have been proven to save lives. The small sample size means the data are not amenable to multiple regression analysis to adjust for confounders. The small sample size raises the possibility of type $\mathrm{b}$ error; however, we still found statistically and clinically significant differences in most of our clinical outcomes of interest.

We believe that differences in comparison were minimised by the use of a standardised mortality ratio to correct for differences between the groups. Statistical comparison of ratios requires a large sample size, which meant that our clinically significant improvement in SMR was not statistically significant, and we cannot therefore definitively conclude that our changes improved survival.

\section{CONCLUSION}

Translation of the Surviving Sepsis Guidelines into our practice was achieved by critical examination of our existing practice, by identification and resolution of a number of key delays, and by intensive education of ED staff on the identification and treatment of patients who have severe sepsis. We believe that our intervention is generalisable to other centres to achieve similar improvements.

Funding Other funders: St Paul's Hospital Foundation.

\section{Competing interests None.}

Ethics approval This study was conducted with the approval of the Providence Health Care REB.

Contributors All authors were involved in designing and performing the study. $\mathrm{RM}, \mathrm{KH}$, RS, RN and DJ contributed to data collection and analysis. RM, RS and PD contributed to preparation and finalisation of the manuscript.

Provenance and peer review Not commissioned; externally peer reviewed.

\section{REFERENCES}

1. Alberti C, Brun-Buisson C, Burchardi $\mathrm{H}$, et al. Epidemiology of sepsis and infection in ICU patients from an international multicentre cohort study. Intensive Care Med 2002; 28:108-21.

2. Angus DC, Linde-Zwirble WT, Lidicker J, et al. Epidemiology of severe sepsis in the United States: analysis of incidence, outcome, and associated costs of care. Crit Care Med 2001:29:1303-10.

3. Levy MM, Fink MP, Marshall JC, et al. 2001 SCCM/ESICM/ACCP/ATS/SIS International Sepsis Definitions Conference. Intensive Care Med 2003;29:530-8.

4. Dellinger RP, Levy MM, Carlet JM, et al. Surviving Sepsis Campaign: international guidelines for management of severe sepsis and septic shock: 2008. Crit Care Med 2008; 36:296-327.

5. Dellinger RP, Carlet JM, Masur $\mathrm{H}$, et al. Surviving Sepsis Campaign guidelines for management of severe sepsis and septic shock. Crit Care Med 2004;32:858-73.

6. de Koning $\mathbf{H}$, Verver JP, van den Heuvel J, et al. Lean six sigma in healthcare. $J$ Healthc Qual 2006;28:4-11.

7. Ulm K. A simple method to calculate the confidence interval of a standardized mortality ratio (SMR). Am J Epidemiol 1990;131:373-5.

8. Mant J. Process versus outcome indicators in the assessment of quality of health care. Int J Qual Health Care 2001;13:475-80. 
9. Shapiro NI, Howell MD, Talmor D, et al. Implementation and outcomes of the Multiple Urgent Sepsis Therapies (MUST) protocol. Crit Care Med 2006;34:1025-32.

10. Kortgen A, Niederprum P, Bauer M. Implementation of an evidence-based "standard operating procedure" and outcome in septic shock. Crit Care Med 2006:34:943-9.

11. Jones AE, Focht A, Horton JM, et al. Prospective external validation of the clinical effectiveness of an emergency department-based early goal-directed therapy protocol for severe sepsis and septic shock. Chest 2007;132:425-32.
12. Carlbom DJ, Rubenfeld GD. Barriers to implementing protocol-based sepsis resuscitation in the emergency department-results of a national survey. Crit Care Med 2007;35:2525-32.

13. Jones AE, Shapiro NI, Roshon M. Implementing early goal-directed therapy in the emergency setting: the challenges and experiences of translating research innovations into clinical reality in academic and community settings. Acad Emerg Med 2007:14:1072-8. 